For reprint orders, please contact: reprints@futuremedicine.com

\title{
Inside the 2016 American Society of Clinical Oncology Genitourinary Cancers Symposium: part 2 - prostate and bladder cancer
}

\author{
Sebastiano Buti', Chiara Ciccarese ${ }^{2}$, Roberto lacovelli², Melissa Bersanelli', \\ Marina Scarpelli3 ${ }^{3}$ Antonio Lopez-Beltran ${ }^{4}$, Liang Cheng ${ }^{5}$, Rodolfo Montironi ${ }^{3}$, \\ Giampaolo Tortora² \& Francesco Massari,6
}

\section{The American Society of Clinical Oncology Genitourinary Cancers Symposium, Moscone West Building, San Francisco, CA, USA, 7-9 January 2016}

The American Society of Clinical Oncology (ASCO) Genitourinary Cancers Symposium, held in San Francisco (CA, USA), from 7 to 9 January 2016, focused on 'patient-centric care: translating research to results'. Every year, this meeting is a must for anyone studying genitourinary tumors to keep abreast of the most recent innovations in this field, exchange views on behaviors customarily adopted in daily clinical practice and discuss future topics of scientific research. This two-part report highlights the key themes presented at the 2016 ASCO Genitourinary Cancers Symposium, with part 1 reporting the main novelties of kidney cancer and part 2 discussing the most relevant issues which have emerged for bladder and prostate tumors.

First draft submitted: 5 April 2016; Accepted for publication: 19 May 2016; Published online: 17 June 2016

\section{Prostate cancer: from hypofractionated radiotherapy to new agents}

The most flourishing section of the symposium for prostate cancer (PC) was about the treatment of localized disease, with a wide output of Phase III studies in the field of radiotherapy (RT).

The RTOG 0415 noninferiority randomized trial, presented by Lee $e$ al al. (Duke University School of Medicine, NC, USA), demonstrated that a hypofractionated RT could be considered for organ confined disease in men with low-risk PC ( $\mathrm{cT} 1-2 \mathrm{a}$, Gleason $\leq 6$, prostate-specific antigen $[P S A]<10)[1]$. In this study, 1092 hormone-naive patients were overall analyzed, with a median follow-up of 5.8 years. The trial met the primary end point $(\mathrm{PE})$ of noninferior disease-free survival (DFS) for the RT schedule with 70 Gy in 28 fractions over 5.6 weeks compared with the standard schedule with 73.8 Gy in 41 fractions over 8.2 weeks (86 vs $85 \%$ 5-year DFS; hazard ratio [HR]: $0.85 ; 95 \%$ CI: 0.64-1.14). Intensity-modulated RT (IMRT) technique was used in $79 \%$ of cases, $3 \mathrm{D}$ conformal RT in $21 \%$. In terms of biochemical recurrence and early adverse events (AEs) the noninferiority was also confirmed, despite a moderate increase in late grade 2 toxicity observed for the hypofractionated schedule.

'Medical Oncology Unit, University Hospital of Parma, Italy

${ }^{2}$ Medical Oncology, Azienda Ospedaliera Universitaria Integrata, University of Verona, Verona, Italy

${ }^{3}$ Section of Pathological Anatomy, Polytechnic University of the Marche Region, School of Medicine, AOU Ospedali Riuniti, Ancona,

Italy

${ }^{4}$ Department of Surgery, Cordoba University Medical School, Cordoba, Spain

${ }^{5}$ Department of Pathology \& Laboratory Medicine, Indiana University School of Medicine, Indianapolis, IN, USA

${ }^{6}$ Division of Oncology, S.Orsola-Malpighi Hospital, Bologna, Italy

*Author for correspondence: fmassari79@gmail.com

\section{KEYWORDS}

- biomarkers • genitourinary cancer $\bullet$ targeted therapy 
A blow has been struck in favor of the hypofractionated RT also from results of the Phase III CHHiP trial, presented by Dearnaley et al. (The Institute of Cancer Research and The Royal Marsden NHS Foundation Trust, London, UK), randomizing 3216 hormone pretreated patients with T1b-T3a PC to receive IMRT standard treatment (74 Gy, 37 fractions, 7.4 weeks) versus hypofractionated IMRT (respectively, 60 Gy, 20 fractions, 4 weeks or 57 Gy, 19 fractions, 3.8 weeks) [2]. The PE was biochemical (PSA) failure, with a noninferiority margin fixed to 5 around $70 \%$ control rate or HR: 1.2 . The 5-year control rate in the control arm was 88.3\% (95\% CI: 86-90\%). The noninferiority was demonstrated for the 60 Gy schedule, with $\mathrm{HR}_{60 / 74}$ : 0.84 (95\% CI: 0.68-1.03); $\mathrm{p}=0.004$, while the analyses were inconclusive for the 57 Gy RT. The comparison of hypofractionated schedules indeed favored 60 Gy in 20 fractions (HR: 1.44; 95\% CI: 1.13-1.82; $\mathrm{p}=0.003$ ), despite a small increase in late bowel side effects compared with 57 Gy in 19 fractions. In conclusion, the CHHiP trial is a landmark study for RT, recommending a modest hypofractionation with $60 \mathrm{~Gy}$ in 20 fractions as a new standard of care for localized PC.

Two further Phase III studies were presented. The RTOG 9601 trial, presented by Shipley WU (Massachusetts General Hospital, Harvard Medical School, MA, USA), randomized patients to receive 24 months bicalutamide or placebo during and after salvage RT following prostatectomy and elevation of PSA. It demonstrated a significant improvement of long-term overall survival (OS; HR: 0.75; 95\% CI: 0.58-0.98; $\mathrm{p}=0.018)$ and a reduced incidence of metastasis and cancer-specific death $(\mathrm{p}<0.001)$ in the arm with antiandrogen therapy [3]. The EORTC trial 22991, presented by M Bolla (Grenoble Alpes University, Centre Hospitalier Universitaire de Grenoble, Radiotherapy Department, Grenoble, France), comparing 6 months of androgen suppression and RT versus RT alone, demonstrated an improvement of biochemical DFS (HR: 0.53; 95\% CI: 0.42-0.67; p < 0.001) and of progression-free survival (PFS; HR: 0.63; 95\% CI: $0.48-0.84$; $\mathrm{p}=0.001$ ) with the addition of medical castration in intermediate- and high-risk localized T1b-cT2aN0M0 prostatic carcinoma, with no persistent detriment on quality of life (QoL) or sexual function [4].

Fewer novelties were reported for the advanced disease, apart from some updates of already published studies. Just after putting an end to the use of zoledronic acid in hormone sensitive PC, a preplanned analysis from the STAMPEDE trial demonstrated a survival advantage for its combination with celecoxib in the hormone naive metastatic disease (HR: 0.78 ; 95\% CI: 0.62-0.99), notwithstanding the futility of adding celecoxib alone to the standard of care. This result, presented by ND James (University of Warwick, Coventry, UK), worthy of further investigation, could provide a valid alternative to upfront chemotherapy for advanced PC [5].

On the other hand, the QoL assessment of the CHAARTED trial (by LJ Patrick-Miller; The University of Chicago Medical Center, IL, USA) previously recommending upfront chemotherapy for metastatic PC, excluded a long-term negative impact of docetaxel respect to androgendeprivation therapy alone [6]. Indeed, despite an initial decrease on treatment (at 3 months), QoL returned to baseline at 12 months, becoming better for patients who underwent chemotherapy versus only androgen-deprivation therapy [7] .

Furthermore, in the intriguing field of genomic sequencing, promising findings were reported by Myers et al. (American Institute for Diseases of the Prostate, VA, USA) about the frequency of $B R C A$ mutations in the advanced disease, reaching $12 \%$ for $B R C A 2$ and $2 \%$ for $B R C A 1$, respectively [8]. On the basis of this evidence, a new class of agents such as PARP inhibitors would probably come on stage for PC in the coming years.

\section{Urothelial cancer: emerging immunotherapies \& molecular profiling}

Two Phase II studies and one Phase Ib study with three different immune checkpoint inhibitors for the treatment of urothelial carcinoma were presented at the symposium.

JH Hoffman-Censits (The Sidney Kimmel Cancer Center at Thomas Jefferson University, PA, USA) reported the results of a Phase II trial with atezolizumab, a fully humanized antibody $(\mathrm{Ab})$ against PD-L1, in platinum-treated locally advanced or metastatic disease (IMvigor 2010 cohort 2 study) [9]. The study included 316 patients (310 evaluable) receiving intravenous (iv.) atezolizumab $1200 \mathrm{mg}$ every 3 weeks until loss of clinical benefit. The primary end point was overall response rate (ORR). PD-L1 expression on tumor-infiltrating immune cells (IC) was assessed with Ventana SP142 immunohistochemistry (IHC). The ORR was 15 and 19\%, 
respectively, according to Response Evaluation Criteria In Solid Tumors (RECIST) version 1.1 (v1.1) per central review and modified (m) RECIST per investigator, with 5\% of complete responses (according to RECIST v1.1). In total, $46 \%$ of patients had tumor burden reduction and it was associated with PD-L1 IHC status. Higher ORR was associated with higher PD-L1 IHC status, albeit responses were seen in all PD-L1 subgroups. Poor baseline prognostic factors did not affect responses to the drug. Durable responses were seen including poor prognostic subgroups with median duration of response not reached, and many not-responding patients experienced stable disease, suggesting that atezolizumab may provide clinical benefit also to these patients. With a median follow-up of 11.7 months, the median PFS was 2.1 and 2.7 months, respectively, according to RECIST v1.1 per central review and mRECIST per investigator. Median OS was 7.9 months and was longer for patients with higher PD-L1 IHC status (11.4 months). Atezolizumab was well tolerated and treatment-related AEs, mostly fatigue, occurred in $69 \%$ of patients, with only $16 \%$ grade 3-4 AE and 5\% grade 3-4 immunerelated AE. Noteworthy for patients with this disease, no renal toxicity was observed.

MD Galsky (The Tisch Cancer Institute, Icahn School of Medicine at Mount Sinai, NY, USA) reported the results of a Phase II study with gemcitabine and cisplatin (GC standard scheme every 21 days) in combination with ipilimumab, a fully humanized Ab against CTLA-4, in chemonaive metastatic urothelial patients or in those that completed perioperative chemotherapy more than 12 months prior to enrolment [10]. The investigators hypothesized that chemotherapy may lead to immunogenic cell death and other immunomodulatory effects, which could subsequently be exploited with the addition of ipilimumab. The patients received two cycles of GC alone followed by four cycles of GC plus ipilimumab (GCIpi) iv. $10 \mathrm{mg} / \mathrm{kg}$ day 1 every 21 days for four cycles. The primary end point was survival rate at 1 year. A total of 36 patients were enrolled, 25\% with ECOG performance status (PS) $=0$ and $58 \%$ with visceral metastases. Toxicity due to chemotherapy was as expected (mostly hematologic), while the most common grade 3-4 immune-related AE were colitis $(6 \%)$, hypophysitis (3\%), hyperthyroidism (1\%) and rash (1\%?). The ORR was $64 \%$ with $14 \%$ of complete responses. Median PFS was 8 months and median OS was 14.6 months, with 1-year OS of $59 \%$. An immune profile revealed that $\mathrm{GC}$ alone had no significant impact on circulating IC subsets, while ipilimumab significantly expanded circulating $\mathrm{CD}^{+}$and $\mathrm{CD}^{+}$ T cells.

AB Apolo (Center for Cancer Research, National Cancer Institute, NIH, MD, USA) reported the results for avelumab, a fully human anti PD-L1 IgG1 Ab, in terms of safety, activity and PD-L1 expression in tumor cells and in tumor infiltrating IC, in patients with metastatic urothelial carcinoma from the JAVELIN Solid Tumor Phase Ib trial [11]. The peculiarity of avelumab, in contrast with other immunecheckpoint inhibitors, is its ability to promote Ab-dependent cell-mediated citotoxicity, as shown in preclinical models [12]. In total, 44 patients with urothelial carcinoma (unselected for PD-L1 expression) that progressed after treatment with at least one platinum-containing regimen for unresectable or recurrent disease were enrolled to receive avelumab iv. $10 \mathrm{mg} / \mathrm{kg}$ every 2 weeks. The PE was not stated. A total of $43 \%$ of patients had PS $=0(43.2 \%)$ and $63 \%$ had visceral metastases. Treatment-related treatmentemergent AEs of any grade occurred in 59\% of patients; those occurring more frequently were grade $1-2$ infusion-related reactions (18\%) and fatigue (16\%). ORR was $16 \%$ with one complete response, disease-control rate (ORR plus stable disease rate) was 59\%; median PFS was 12 weeks (47\% of patients alive and progression free at this time point). PD-L1 expression was evaluable in 32 patients and there was a trend towards higher ORR and prolonged PFS rate at 12 weeks in patients with PD-L1-positive tumors [13].

In 2014 the Cancer Genome Atlas Research Network reported, as part of The Cancer Genome Atlas (TCGA) project, an integrated analysis of 131 urothelial carcinomas to provide a comprehensive landscape of molecular alterations of these tumors [14]. The same authors (TCGA Bladder Cancer Working Group, several US and Canadian institutions) then reported at the symposium the comprehensive characterization of 412 muscle chemotherapy-naive invasive urothelial carcinomas as final analysis of TCGA project [15]. Tumors were analyzed for DNA copy number variants, whole exome sequencing, DNA methylation, mRNA, miRNA and (phospho-) protein expression, transcript splicing, gene fusions, viral integration, pathway perturbation, clinical correlates and histopathology. The 
investigators confirmed a high overall somatic mutation rate $(8.0 / \mathrm{Mb})$ as reported in the previous analysis and interestingly high mutation burden was associated with improved outcome ( $p=0.0004)$. There were 54 significantly mutated genes, increased from 32 in the original report. TP53 remained the most commonly mutated gene, and chromatin-modifying genes were also frequently mutated. $K R A S, E R B B 2, R B 1$ and $E L F 3$ genes showed significant increased frequencies of mutation. One-hundred and sixty-seven genes were silenced by promoter DNA hypermethylation in at least 5\% of tumors. Several mutated genes were also epigenetically silenced at various frequencies (ZNF773, CDKN2A, FAT1, $C A S P 8)$. These findings provide a robust basis for functional studies to further understand the biology of bladder cancer, favoring the development of more tailored targeted therapies.

\section{Conclusion}

In PC, only confirmatory studies have been reported in nonmetastatic disease. The RTOG
9601 trial confirms that radiotherapy plus bicalutamide is better than radiotherapy alone but the standard of care for intermediate- as well as for high-risk disease is still the LH-RH analogue. No new evidence has been reported about duration of therapy, which remains a major issue in clinical practice.

Bladder cancer is still a new field in immunotherapy, mainly for the patients who progress after a platinum based treatment and with an increasing amount of biological knowledge and high expectation for the ongoing Phase III trials.

\section{Financial \& competing interests disclosure}

The authors have no relevant affiliations or financial involvement with any organization or entity with a financial interest in or financial conflict with the subject matter or materials discussed in the manuscript. This includes employment, consultancies, honoraria, stock ownership or options, expert testimony, grants or patents received or pending, or royalties.

No writing assistance was utilized in the production of this manuscript.

\section{References}

1 Lee WR, Dignam JJ, Amin M et al. NRG Oncology RTOG 0415: a randomized Phase III non-inferiority study comparing two fractionation schedules in patients with low-risk prostate cancer. J. Clin. Oncol. 34(Suppl. 2S), Abstract 1 (2016).

2 Dearnaley DP, Syndikus I, Mossop H et al. Comparison of hypofractionated high-dose intensity-modulated radiotherapy schedules for prostate cancer: RESULTS from the Phase III randomized $\mathrm{CHHiP}$ trial (CRUK/06/016). J. Clin. Oncol. 34(Suppl. 2S), Abstract 2 (2016).

3 Shipley WU, Pugh SL, Lukka HR et al. NRG Oncology/RTOG 9601, a Phase III trial in prostate cancer patients: anti-androgen therapy (AAT) with bicalutamide during and after salvage radiation therapy (RT) following radical prostatectomy (RP) and elevated PSA. J. Clin. Oncol. 34(Suppl. 2S), Abstract 3 (2016).

4 Bolla M, Van Den Bergh ACM et al. EORTC trial 22991: results of a Phase III study comparing 6 months of androgen suppression and irradiation versus irradiation alone for localized T1b-cT2aN0M0 prostate cancer. J. Clin. Oncol. 34(Suppl. 2S), Abstract 22 (2016).

5 James ND, Sydes MR, Clarke NW et al. Celecoxib with or without zoledronic acid for hormone-naive prostate cancer: survival results from STAMPEDE (NCT002684476). J. Clin. Oncol. 34(Suppl. 2S), Abstract 162 (2016).

6 Patrick-Miller LJ, Chen YH, Carducci MA et al. Quality of life (QOL) analysis from E3805, chemohormonal androgen ablation randomized trial (CHAARTED) in prostate cancer (PrCa). J. Clin. Oncol. 34(Suppl. 2S), Abstract 286 (2016).

7 Scher HI, Graf R, Louw J et al. Single CTC characterization to identify phenotypic and genomic heterogeneity as a mechanism of resistance to $\mathrm{AR}$ signaling directed therapies (AR Tx) in mCRPC patients. J. Clin. Oncol. 34(Suppl. 2S), Abstract 163 (2016).

8 Myers CE, Feldman R, Abbott BL et al. Frequency of $B R C A$ mutations and cooccurring alterations in prostate cancer. J. Clin. Oncol. 34(Suppl. 2S), Abstract 289 (2016).

9 Hoffman-Censits JH, Grivas P, Van Der Heijden MS et al. IMvigor 210, a Phase II trial of atezolizumab (MPDL3280A) in platinum-treated locally advanced or metastatic urothelial carcinoma (mUC). J. Clin. Oncol. 34(Suppl. 2S), Abstract 355 (2016).

10 Galsky MD, Hahn NM, Albany C et al. Phase II trial of gemcitabine + cisplatin + ipilimumab in patients with metastatic urothelial cancer. J. Clin. Oncol. 34(Suppl. 2S), Abstract 357 (2016).

11 Apolo AB, Infante JR, Hamid O et al. Safety, clinical activity, and PD-L1 expression of avelumab (MSB0010718C), an anti-PD-L1 antibody, in patients with metastatic urothelial carcinoma from the JAVELIN Solid Tumor Phase Ib trial. J. Clin. Oncol. 34(Suppl. 2S), Abstract 367 (2016).

12 Boyerinas B, Jochems C, Fantini M et al. Antibody-dependent cellular cytotoxicity activity of a novel anti-PD-L1 antibody avelumab (MSB0010718C) on human tumor cells. Cancer Immunol. Res. 3(10), 1148-1157 (2015).

13 Faltas B, Bhinder B, Beltran $\mathrm{H}$ et al. Generating a neoantigen map of advanced urothelial carcinoma by whole exome sequencing. J. Clin. Oncol. 34(Suppl. 2S), Abstract 354 (2016).

14 Weinstein JN, Akbani R, Broom BM et al. Comprehensive molecular characterization of urothelial bladder carcinoma. Nature 507(7492), 315-322 (2014).

15 Lerner SP, Kim J, Kwiatkowski DJ et al. Comprehensive characterization of 412 muscle invasive urothelial carcinomas: final analysis of The Cancer Genome Atlas (TCGA) project. J. Clin. Oncol. 34(Suppl. 2S), Abstract 405 (2016). 